\title{
MODELING OF ENTROPY PRODUCTION AND SELF-ORGANIZATION OF DECOMPOSING PACS 66.30.Qa, 72.15.Eb METALLIC ALLOY UNDER HIGH CURRENT DENSITY
}

\begin{abstract}
The synergy of the decomposition and electromigration in binary alloys under a very strong electric current is analyzed in the frame of the entropy production rate approach and simulated by the Monte Carlo method. The morphology evolution and the Joule heating rate time behavior depend on what is fixed during the electromigration - current or voltage.

Keywords: electromigration, alloy, decomposition, Joule heating, entropy production, electron wind, Monte Carlo method, structure.
\end{abstract}

\section{Introduction}

The current in a metal alloy causes at least two effects - (1) the emission of Joule heat (and the corresponding rate of entropy production $\dot{S}^{\text {Joule }}[1]$ due to the scattering of electrons by defects), (2) electromigration of atoms of both components of the alloy under the influence of an electron wind [2-5]. The second effect is also related to the entropy production $\dot{S}^{\text {atomic }}$; however, it requires a longer time period and high current densities for its fixation. If the alloy is heterogeneous (e.g., it is a mixture of two phases), higherorder cross-effects also occur. For example, the difference in the specific resistances of the phases leads to the locally heterogeneous heat generation and, as a consequence, to local temperature gradients. These gradients cause the thermomigration and, in some cases, can lead to "anticoalescence" [6]. Here, we will not go so far, but restrict ourselves to only two main processes - Joule heat and electromigration. However, at the same time, we will investigate the interdependence of these processes with the process of diffusion decomposition of the alloy. In addition, we will discuss whether the often discussed principles of entropy production extremality and other principles of extremality are implemented [7-10]. In this case, it will be important to clearly fix, on which set the extremum is looking for. One thing is the extremum of entropy production relative to the variations of the spatial distribution of potentials and the current den-

(C) A.M. GUSAK, S.V. MARCHENKO, V.V. TURLO, A.O. BOGATYREV, 2017

ISSN 2071-0194. Ukr. J. Phys. 2017. Vol. 62, No. 12 sity at a fixed atomic distribution. Another thing is the extremum (if it exists!) of the entropy production due to variations of the spatial distribution of atoms. The entropy production goes to the extremum alongside the evolution of the morphology of the alloy in the course of the time.

Due to the miniaturization of elements of integrated circuits, the density of currents in them has significantly increased. In some cases, this leads to the formation of ordered structures. An example of the ordering of the two-phase structure of a solder along the lines of the current is shown in Fig. 1 (see, e.g., a monograph [11]). Since this structure is an open system in the field of external currents, it can be interpreted as an example of self-organization in open systems, which is associated with the extreme properties of entropy production. In addition, the minimization of entropy production, as suggested by K.-N. Tu [2], may be the cause for the so-called gradient forces acting on a nanopore under the conditions of a sharply heterogeneous distribution of currents in a chip. Namely, it is empirically noted that the pores tend to exit the region of the congestion of the current lines ("current crowding" areas). K.-N. Tu explains this as the aspiration to reduce the energy dissipation.

Consider the stationary distributions of the current density $\mathbf{j}(\mathbf{r})$ and electrical potential $\varphi(\mathbf{r})$ in a non-uniform (e.g., two-phase) conductive alloy with a known spatial distribution of the electrical conductivity $\sigma(\mathbf{r})$. The local form of Ohm's law for the current density $(\mathbf{j}=\sigma(\mathbf{r}) \mathbf{E}=-\sigma(\mathbf{r}) \operatorname{grad} \varphi(\mathbf{r}))$ together with the local form of Kirchhoff's first law (i.e., the 


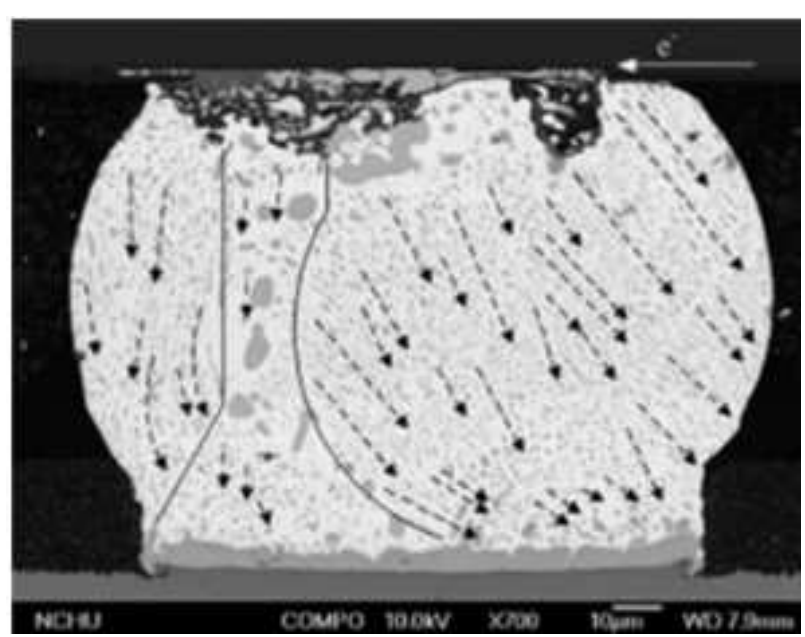

Fig. 1. Typical morphology of a two-phase solder under the influence of electromigration. The arrows indicate the local current direction [11]

law of conservation of the charge and the condition of stationarity, $\operatorname{div} \mathbf{j}=0$ ) lead us to the well-known equation for a stationary potential distribution:

$\operatorname{div}(\sigma(\mathbf{r}) \operatorname{grad} \varphi(\mathbf{r}))=0$.

As G. Kirchhoff noticed long before, by introducing the concept of entropy and its production (see [1]), Eq. (1) can alternatively be interpreted as a minimum energy dissipation condition (Joule heat). In the approximation of the temperature uniformity (which is sufficiently well performed in metal samples under a current), it can be reformulated as a minimum condition for the part of entropy production that is not related to the redistribution of components, i.e., the production of entropy with a fixed distribution of conductivity (with a fixed distribution of alloy components) and under fixed boundary conditions. In addition, this property can be used as a means for solving Eq. (1). At the same time, solving Eq. (1) by minimizing the functional of the total Joule heat may prove to be most productive for a numerical solution of the three-dimensional problem (1) with a complex conductivity distribution.

Briefly, we recall the basic ideas of Joule heat extremalization. The production of entropy by the Joule heating of a temperature-homogeneous system with volume $V$,

$\dot{S}^{\text {Joule }}=\iiint_{V} \frac{\mathbf{j E}}{T} d V=\frac{1}{T} \iiint_{V} \sigma(\mathbf{r})(\nabla \varphi)^{2} d V$, is a function of the spatial distribution of the potential $\varphi(\mathbf{r})$. A variation of this functional equals

$$
\begin{aligned}
& \delta \dot{S}^{\text {Joule }}=\frac{2}{T} \iiint_{V} \sigma(\mathbf{r})(\nabla \varphi) \nabla(\delta \varphi) d V= \\
& =\oint_{\Sigma(V)} \sigma(\mathbf{r}) \frac{\partial \varphi}{\partial n} \delta \varphi d V-\frac{2}{T} \iiint_{V} \nabla(\sigma(\mathbf{r})(\nabla \varphi)) \delta \varphi d V .
\end{aligned}
$$

Here, we consider the following boundary conditions on the limiting surface for the volume $V-\Sigma(V)$ : (1) on two boundaries, the potentials are fixed (voltage is given at the ends of the sample between the "cathode" and "anode"), so that the variation of the potential at these boundaries is equal to zero: $\left.\delta \varphi\right|_{\Sigma}=$ $0,(2)$ on the rest of the boundary, the metal sample is bounded by dielectrics, i.e., at each point of such boundaries, the normal component of the current density is equal to zero. Hence, the derivative of the potential along the normal to the surface equals zero, $\left.\frac{\partial \varphi}{\partial n}\right|_{\Sigma}=0$. In the case of Born-Karman's periodic boundary conditions on the lateral boundaries, the normal component of the current, say, on the "upper" boundary, may not be equal to zero, but the current that flows through the upper boundary will be fully offset by the current entering the system through the bottom boundary in accordance with periodic boundary conditions. For the above reasons, the first integral (on a surface) on the right-hand side of Eq. (3) is zero. So,

$\delta \dot{S}^{\text {Joule }}=-\frac{1}{T} \iiint_{V} \nabla(\sigma(\mathbf{r})(\nabla \varphi)) \delta \varphi d V$.

The extremum of a functional means the equality of the first variation of a functional with an arbitrary small variation of a function to zero. This means that the integral expression in Eq. (4), $\nabla(\sigma(\mathbf{r})(\nabla \varphi))$, is zero under the condition of entropy production extremality. Thus, Eq. (1) can be interpreted, indeed, as the Euler-Lagrange equation of the variational problem for the total rate of heat generation and the corresponding part of entropy production (in the approximation of the temperature field uniformity). The extremal is sought in a class of functions $\varphi(\mathbf{r})$ with the before-specified boundary conditions and the given distribution of conductivity $\sigma(\mathbf{r})$, i.e., with a given distribution of atoms in the alloy.

ISSN 2071-0194. Ukr. J. Phys. 2017. Vol. 62, No. 12 
Under these conditions, Eq. (1) is a consequence of the extremality of entropy production. In other words, the system distributes currents and potentials within itself so that the dissipation of energy is extremal. The fact that this extremum is a minimum is guaranteed by the positive conductivity in functional (2) (details are given in the next paragraph).

In this paper, we propose an atomistic model of the distributions of currents and potentials, which is then used to simulate simultaneously the decomposition of an unstable alloy and the electromigration of atoms in it. We wonder whether any extremal principles work at the level of a distribution of phases in a two-phase system, rather than currents and potentials. At the intuitive level, one can expect that a change in the current morphology should somehow correlate with that part of the production of entropy, which is associated with Joule heat. For example, if we assume that the system is trying to reduce the energy dissipation, then, at a given current, it will try to reduce the resistance (i.e., to evolve toward the parallel phase connection) and, at a fixed voltage, to increase the resistance (i.e., to evolve toward the successive connection of phases). We will see below that our intuition is justified only partially!

\section{Discrete Atomistic}

\section{Model of Distribution of Currents}

Consider the discrete atomistic model of binary alloy, which is based on the approximation of a regular solid solution, say, with a face-centered cubic lattice (or in the two-dimensional case - with a triangular one). The alloy is characterized by a positive mixing energy $E^{\text {mix }}=V_{A B}-\frac{V_{A A}+V_{B B}}{2}>0$. At a fairly low temperature $T<\frac{Z E^{\text {mix }}}{2 k_{\mathrm{B}}}(Z=12$ for an FCC lattice, and $Z=6$ for a triangular lattice), the system has a two-phase area and can decompose itself. Here, we shall consider the decomposition simultaneously with the electromigration caused by the electric current with given potentials on the anodic and cathodic surfaces. It will be simpler to assume that the current in the alloy goes from node to node through a connecting "internode conductor". Let I, In be the indices of an arbitrarily selected node and one of its $Z$ neighbors (each of the indices I and In actually represents three indices in three axes). By $\sigma[\mathrm{I}]$ and $\sigma[\operatorname{In}]$, we denote the conductivities corresponding to the filling of nodes I and In. Conductance $\sigma[\mathrm{I}]$ attributed to the node is equal to $\sigma_{A}$ or $\sigma_{B}$, depending on the filling of the site [11].

The contact between the nodes is attributed to the average proportional conductivity

$\sigma[\mathrm{I}, \mathrm{In}]=\frac{2 \sigma[\mathrm{I}] \sigma[\mathrm{In}]}{\sigma[\mathrm{I}]+\sigma[\mathrm{In}]}$.

For the specific resistance, this means the arithmetic mean (which is logical for the sequential connection of the two nodes)

$\rho[\mathrm{I}, \mathrm{In}]=\frac{\rho[\mathrm{I}]+\rho[\mathrm{In}]}{2}$.

The current density in the direction from node I to the neighboring node In, $j[\mathrm{I} \rightarrow \mathrm{In}]$, in our discrete scheme is given by the relation

$j[\mathrm{I} \rightarrow \mathrm{In}]=-\sigma[\mathrm{I}, \mathrm{In}] \frac{\varphi[\mathrm{In}]-\varphi[\mathrm{I}]}{d}$

(here, $d$ is the interatomic distance; in the case of a FCC lattice, $d=a / \sqrt{2}$ ). As is known, the flux divergence is defined as the limit of the ratio of the flux through the constricted surface to the volume inside this surface. In our discrete scheme, the constriction stops at the level of a Wigner-Seitz cell, which is a polyhedron with atomic volume $\Omega$ and (in the case of a FCC lattice) $Z=12$ faces with the same area $S_{1}$ :

$$
\begin{aligned}
& \operatorname{div} \bar{j}(\mathrm{I})=\frac{\oint_{\Sigma(\Omega)} j_{n} d S}{\Omega}=\frac{\sum_{\mathrm{In}=1}^{Z} j[\mathrm{I} \rightarrow \mathrm{In}] S_{1}}{\Omega}= \\
& =\frac{S_{1}}{\Omega} \sum_{\mathrm{In}=1}^{Z} \sigma[\mathrm{I}, \mathrm{In}](\varphi[\mathrm{I}]-\varphi[\mathrm{In}]) .
\end{aligned}
$$

Thus, the discrete analog of the differential equation (1) is the system of algebraic equations

$$
\sum_{\mathrm{In}=1}^{Z} \sigma[\mathrm{I}, \mathrm{In}](\varphi[\mathrm{I}]-\varphi[\operatorname{In}])=0, \quad \mathrm{I}=1, \ldots, N
$$

In this case, the potentials on the cathodic and anodic nodes are given. The equilibrium of the normal components of the current density at the lateral surfaces equals zero either by periodic boundary conditions or by the automatic zeroing of the conductivity at the boundary with the dielectric node:

$\sigma\left[\mathrm{I} \mathrm{In}^{\text {dielectric }}\right]=\frac{2 \sigma[\mathrm{I}] 0}{\sigma[\mathrm{I}]+0}$. 
An analog of functional (2) in our discrete scheme is a function of the values of the potential in all nodes of the system:

$\dot{S}^{\text {Joule }}(\varphi[1], \ldots, \varphi[N]) \propto$

$\propto \frac{1}{T} \sum_{\mathrm{I}=1}^{N} \sum_{\mathrm{In}=1}^{Z} \frac{\sigma[\mathrm{I}, \mathrm{In}](\varphi[\mathrm{I}]-\varphi[\mathrm{In}])^{2}}{d^{2}} \Omega$.

The minimum conditions are formulated as follows:

$$
\begin{aligned}
& \frac{\partial \dot{S}^{\text {Joule }}(\varphi[1], \ldots, \varphi[N])}{\partial \varphi[\mathrm{I}]}=0, \quad \mathrm{I}=1, \ldots, N, \\
& \frac{\partial^{2} \dot{S}^{\text {Joule }}(\varphi[1], \ldots, \varphi[N])}{\partial \varphi[\mathrm{I}]^{2}}>0 .
\end{aligned}
$$

Substituting expression (11) into the minimal conditions (12), we obtain

$$
\begin{aligned}
& \sum_{\operatorname{In}=1}^{Z} \sigma[\mathrm{I}, \operatorname{In}](\varphi[\mathrm{I}]-\varphi[\operatorname{In}])=0, \\
& \sum_{\operatorname{In}=1}^{Z} \sigma[\mathrm{I}, \operatorname{In}]>0, \quad \mathrm{I}=1, \ldots, N .
\end{aligned}
$$

In other words, the condition of minimal Joule heat yields the basic system of equations (9).

Thus, the minimization of the entropy production for a stationary distribution of currents with a fixed distribution of conductivity and fixed voltages at the ends of the conductor is strictly fulfilled and confirms the principle of minimal entropy production in the stationary states of open linear systems. This principle [7] is proved only in the case of constant kinetic coefficients. In a more general case, a change of the entropy production with time is divided into two parts: the first one, $\frac{d_{X} \sigma}{d t}$, is associated with changes in driving forces, and the second part, $\frac{d_{J} \sigma}{d t}$, with changes of fluxes:

$\frac{d \sigma}{d t}=\frac{d_{X} \sigma}{d t}+\frac{d_{J} \sigma}{d t}$.

For the first term, the non-positivity is proved $\left(\frac{d_{X} \sigma}{d t}<=0\right)$. For the second term, such a property is not proved and, in general, is unfair.

It should be noted that there are still many discrepancies regarding the principles of entropy production extremality. In fact, one should distinguish cases where the entropy production goes to a minimum and, when it is, tends to maximum by contrast. If the thermodynamic system is closed, i.e., it is under homogeneous external conditions (either adiabatic ones or in a spatially homogeneous thermostat), it tends to equilibrium that corresponds to the extremum of any thermodynamic potential (the maximum of entropy if the system is isolated, the minimum of Helmholtz's free energy; if the temperature of the thermostat and the volume of the system are fixed, the minimum free energy of Gibbs; if the temperature of the thermostat and the external pressure are fixed). In all these cases, the production of entropy in an extreme state (in this case, it is an equilibrium one) is zero. If the thermodynamic system is open, i.e., it is under heterogeneous, but constant in time external conditions (e.g., different, but constant temperatures or chemical, or electrical potentials within the limits), then it will never come to equilibrium, but, most likely, will be in a stationary state, which is characterized by constant fluxes (an alternative is the oscillatory regime). In the simplest case, this stationary state corresponds to a minimum of entropy production. This property has no direct relation to the problem of choosing the path of evolution. To the final state (equilibrium or stationary), the system can move by different ways. Many physicists "believe" that the system chooses usually the path that corresponds to the maximum entropy production.

This hypothesis sounds quite plausible, but it is not always strictly valid $[8,9]$. We give an opportunity to answer all these questions to the model system itself. It is known that the electromigration in a decomposing alloy can change the morphology of the system. Our task is to find out how, in this case, the production of entropy changes, especially the part (main) that is associated with Joule heat. Additional sources of entropy production (if temperature gradients are neglected) are the electromigration and decomposition. The ratio of the entropy production due to the electromigration to that due to the Joule heat is

$\frac{\frac{1}{\Omega} \frac{D}{k_{\mathrm{B}} T}(\Delta Z e E)^{2} / T}{\sigma E^{2} / T}=\frac{D(\Delta Z e)^{2}}{\Omega k_{\mathrm{B}} T \sigma}$.

The realistic values of the diffusion coefficients, effective charges, and conductivities give a very small

ISSN 2071-0194. Ukr. J. Phys. 2017. Vol. 62, No. 12 
value of this ratio (less than a millionth part), i.e., the contribution of the electromigration to the production of entropy can be neglected. As for the chemical part (the production of entropy due to the decomposition), this case is more complicated and depends on the mechanism and the stage of the decomposition. An analytical theory of the early stages of spinodal decay in the presence of the electromigration will be outlined elsewhere.

\section{Finding the Distribution of Voltages and Currents in the Fixed Distribution of Components}

In the general case, the problem of alloy self-organization in the presence of a strong current is very complex, since it involves a feedback: the electron wind influences differently the migration of atoms of $\mathrm{A}$ and $\mathrm{B}$ sorts and thus changes the spatial distribution of atoms between the nodes, and this distribution determines, in turn, the distribution of potentials, current, and electron wind. Therefore, strictly speaking, it is necessary to redefine the distribution of potentials after each diffusion jump, i.e., to solve an optimization problem. It is technically very difficult to do. So, we calculate the potentials by the Monte Carlo procedure. We tested three methods:

(1) a simple iterative method,

(2) a gradient descent method with a constant step,

(3) fast gradient descent method with an optimized step.

\subsection{Simple iterative method}

The system of equations (9) can be represented as follows:

$$
\varphi[\mathrm{I}]=\frac{\sum_{\mathrm{In}=1}^{Z} \sigma[\mathrm{I}, \mathrm{In}] \varphi[\mathrm{In}]}{\sum_{\mathrm{In}=1}^{Z} \sigma[\mathrm{I}, \mathrm{In}]}, \quad \mathrm{I}=1, \ldots, N .
$$

The idea of the iterative method is that the left-hand side is treated as the next iteration, while the righthand side is as a current one:

$$
\varphi^{\text {iter }+1}[\mathrm{I}]=\frac{\sum_{\mathrm{In}=1}^{Z} \sigma[\mathrm{I}, \mathrm{In}] \varphi^{\text {iter }}[\mathrm{In}]}{\sum_{\mathrm{In}=1}^{Z} \sigma[\mathrm{I}, \mathrm{In}]}, \quad \mathrm{I}=1, \ldots, N
$$

The reassignment according to relation (14) occurs for all nodes except the cathodic and anodic ends, on which the potentials are considered to be given. The initial iteration is traditionally adopted by a linear function that acquires fixed values at the anodic and cathodic ends: $\varphi^{0}(I)=\varphi^{\text {cathode }}+\frac{x}{L_{x}}\left(\varphi^{\text {anode }}-\right.$ $\left.-\varphi^{\text {cathode }}\right)$. The disadvantage of this method is that the iterative procedure does not always ensure convergence. In the case of the usage of the method for the random distribution of atoms, a minimum of Joule heat is achieved fairly rapidly, but there is a further increase in the parameter $P$ [see formula (15)], which indicates a deviation of the values of the nodal potentials from the real values, and, respectively, the divergence of the iteration procedure.

\subsection{Gradient descent method with constant step}

The gradient descent method is based on the quadratic minimization theorem proved above. We have

$P=\sum_{\mathrm{I}=1}^{N} \sum_{\mathrm{In}=1}^{Z} \sigma[\mathrm{I}, \mathrm{In}](\varphi[\mathrm{I}]-\varphi[\mathrm{In}])^{2}$.

When choosing a constant step of the gradient descent at each step, the potentials at each node are redefined by the formula

$\varphi[\mathrm{I}]=\varphi[\mathrm{I}]-2 \lambda \sum_{\mathrm{In}=1}^{Z} \sigma[\mathrm{I}, \mathrm{In}](\varphi[\mathrm{I}]-\varphi[\mathrm{In}])$.

Here, in relation (16), the step $\lambda$ has an inverse to the conductivity units, i.e. $[\Omega m]$.

To verify the efficiency of the gradient descent method, a model two-dimensional sample with a triangular lattice, whose nodes form a block structure, was used: up to half the length, the sample is filled with atoms of the same sort; after, with atoms of another sort. Using the gradient descent method can eliminate the lack of the previous algorithm, namely, the possible divergence of successive approximations.

\subsection{Fast gradient descent method}

The idea of the method is the same, but there is a onedimensional optimization of the change of $P$ with a step $\lambda$ (i.e., the first derivative of the change of $P$ with respect to the step is zero, and the second derivative 
is greater than zero). In this case, the optimal step is determined by the formula

$\lambda^{\mathrm{opt}}=$

$=\frac{\sum_{\mathrm{I}} \sum_{\mathrm{In}} \sum_{\operatorname{Inb}} \sigma[\mathrm{I}, \mathrm{In}] \sigma[\mathrm{I}, \operatorname{Inb}](\varphi[\mathrm{I}]-\varphi[\mathrm{In}])(\varphi[\mathrm{I}]-\varphi[\mathrm{Inb}])}{2 \sum_{\mathrm{I}} \sum_{\mathrm{In}} \sigma[\mathrm{I}, \mathrm{In}]\left(\sum_{\mathrm{Inb}} \sigma[\mathrm{I}, \mathrm{Inb}](\varphi[\mathrm{I}]-\varphi[\mathrm{Inb}])\right)^{2}}$.

Given that the iteration procedure congerges with a computation accuracy sufficient for our modeling, and the optimization procedure has a lower convergence rate for a large number of particles, the iterative approach was chosen for our calculations.

\section{Investigation of the Time \\ Dependence of the Morphology, Resistance, and Entropy Production in a Decomposing Alloy}

The first results for a two-dimensional model (triangular lattice) in the case of a fixed current were obtained by the iterative method in [12] with the participation of two authors of this article (A.M. Gusak, V.V. Turlo). Here, we has complicated the problem, by considering two alternatives for the potential (fixed current and fixed voltage) and two alternative boundary conditions for the electromigration of atoms (zero fluxes at the limits and periodic boundary conditions). A fixed voltage is provided by simply fixing the potentials on the left and right boundaries of the sample. The most convenient way to fix a current is to renormalize the potential at all points of the scheme. In the case of zero fluxes at the boundaries, the segregation of almost pure components within the boundaries (as observed) can be expected. An alternative assignment of periodic boundary conditions physically means simply allocating a small part of the sample in the region of quasistationary flows of atoms outside the segregation regions. A simulation in a three-dimensional case is also included. The Monte Carlo method was used for the exchange mechanism in a decomposing binary alloy. A standard Metropolis algorithm was used with the following modification: the change in the energy at the exchange of atoms $A$ in the node In and $B$ in the site I included the electromigration term

$\Delta E^{q}=\left(Z_{A}-Z_{B}\right) e(\varphi[\mathrm{I}]-\varphi[\operatorname{In}])$,
$Z_{A}, Z_{B}$ are the effective charges of components. The task includes a characteristic size

$$
l^{\mathrm{el}}=\frac{k_{\mathrm{B}} T}{\Delta Z e(\rho j)^{\text {average }}}=L \frac{k_{\mathrm{B}} T}{\Delta Z e\left(\varphi^{\text {anode }}-\varphi^{\text {cathode }}\right)},
$$

where $L$ is the complete longitudinal length of a sample.

While the characteristic size of the heterogeneity of the decomposing alloy is less than $l^{\mathrm{el}}$, the expected impact of the electromigration on the redistribution of components is small. Therefore, it can be expected that, at the initial stage of the decomposition, the effect of the electromigration will be relatively small, and all Joule heat changes will be determined firstly by simply increasing the characteristic length of the heterogeneity (e.g., increasing the size of inclusions of the second phase and the corresponding decrease in their number), which means a decrease in the resistance (see below).

When constructing a two-dimensional model, some model parameters remote from reality (especially a huge current density) were used, which allow tracing the evolution of the structure for a reasonable machine time: the size of the sample $N_{X}=600, N_{Y}=300$, $V_{A A} / k_{\mathrm{B}} T=V_{B B} / k_{\mathrm{B}} T=-1.087, V_{A B} / k_{\mathrm{B}} T=$ $=-0.181$, conductivity of components $A$ and $B$, $\sigma_{A}=6 \times 10^{7} \Omega^{-1} \cdot \mathrm{m}^{-1}, \sigma_{B}=6 \times 10^{6} \Omega^{-1} \cdot \mathrm{m}^{-1}$ respectively, $\Delta Z e=30 \times 10^{-19} \mathrm{C}$, interatomic distance $a=2.55 \times 10^{-10} \mathrm{~m}$.

\subsection{Fixed current \\ in a two-dimensional sample}

\subsubsection{Zero component fluxes at boundaries (phase segregation at the anodic and cathodic ends)}

A typical picture of the evolution of a morphology in the case of a fixed current and a zero flux of components on the cathode and anode is shown in Fig. 2. The system shows the competition of two trends: to the sequential connection as a result of the segregation on the electrodes and to the parallel connection in the inner part of the sample.

The intensity of the Joule heat generation in the case of a fixed current is obviously proportional to the total resistance and, therefore, repeats the form of the dependence of Fig. 3, which shows the typical time dependence of the total resistance.

ISSN 2071-0194. Ukr. J. Phys. 2017. Vol. 62, No. 12 


\subsubsection{Periodic boundary conditions for components}

The nonmonotonicity of the time dependence of the Joule heat generation is obviously related to the segregation of the phases on the cathode and the anode and, as a result, to the increase of the fraction of the serial connection in the two-phase alloy. In order to notice the desired trend in its pure form, it is necessary to get rid of the effects of areas of the electromigration segregation. To do this, of course, you can simply trim the extreme areas, but we will choose another way: without changing the boundary conditions for currents and potentials, we introduce new boundary conditions for the components on the boundaries; instead of the zero fluxes of components, we introduce periodic boundary conditions for the components on the cathode and the anode. The results are presented in Figs. 4 and 5.

\subsection{Fixed voltage}

in a two-dimensional sample. Zero component flows at the boundaries

Next, we substitute the voltage for the current, by expecting that the structure strives to a consistent con-

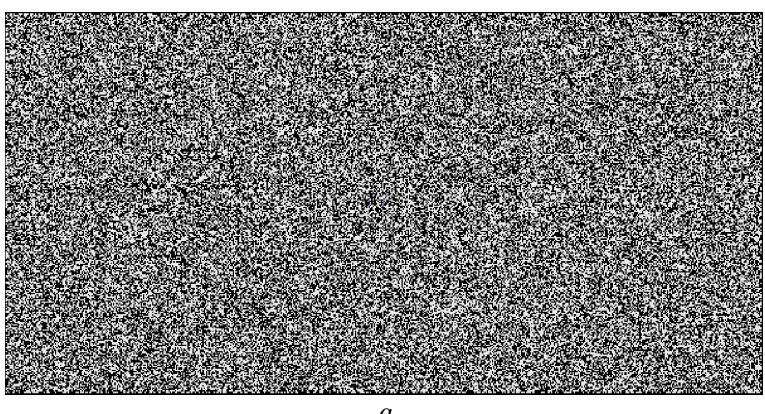

$a$

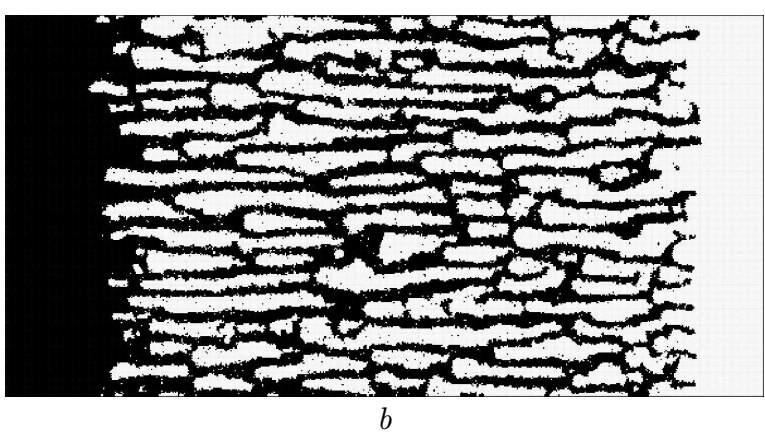

Fig. 2. Decomposition of an alloy during the transmission of a fixed current (triangular lattice, zero component fluxes at the boundaries); initial state (a), after 5000 Monte Carlo steps (b); $V_{A A} / k_{\mathrm{B}} T=V_{B B} / k_{\mathrm{B}} T=-1.087, V_{A B} / k_{\mathrm{B}} T=-0.181$, $l^{\mathrm{el}} / a=0.18$

ISSN 2071-0194. Ukr. J. Phys. 2017. Vol. 62, No. 12

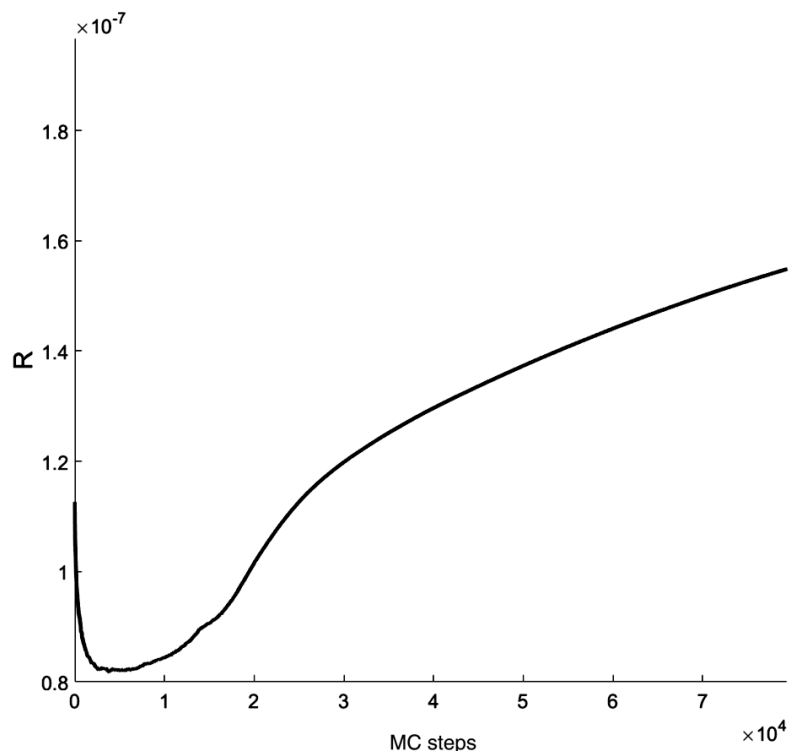

Fig. 3. Typical time dependence of the total resistance with a fixed total current and the zero flows of components at the boundaries. $V_{A A} / k_{\mathrm{B}} T=V_{B B} / k_{\mathrm{B}} T=-1.087, V_{A B} / k_{\mathrm{B}} T=$ $=-0.181, l^{\mathrm{el}} / a=0.18$

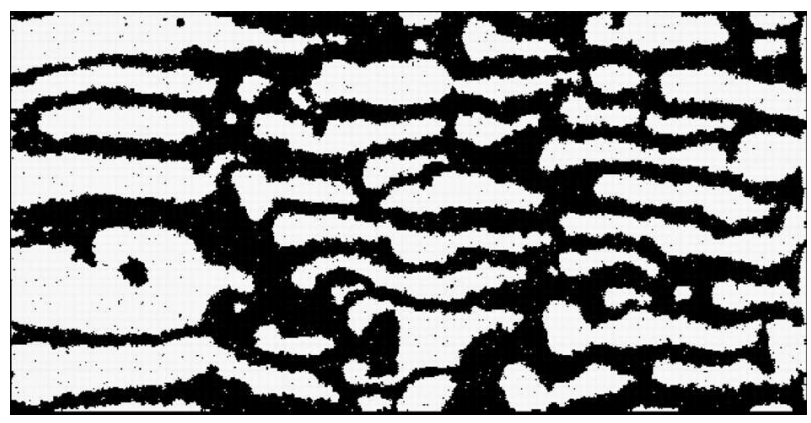

Fig. 4. Structure of a 2D alloy with fixed current and under periodic boundary conditions in atomic fluxes (after 75000 $\mathrm{MC}$ steps, the remaining parameters correspond to the abovepresented ones)

nection. (Our initial idea was that the system strives always to reduce the energy dissipation. Since, at a given voltage, the power of the Joule heat generation is equal to $U^{2} / R$, we expected an increase in the resistance, which the system can provide, by forming the structure of a serial connection). As can be seen in Fig. 6, the situation is somewhat more complicated. The structure of the parallel connection is not really formed, unlike the previous cases. In addition, the tendency to a serial connection really manifests itself, at least for a fairly large voltage, but the resistance of the sample at first still falls and begins 

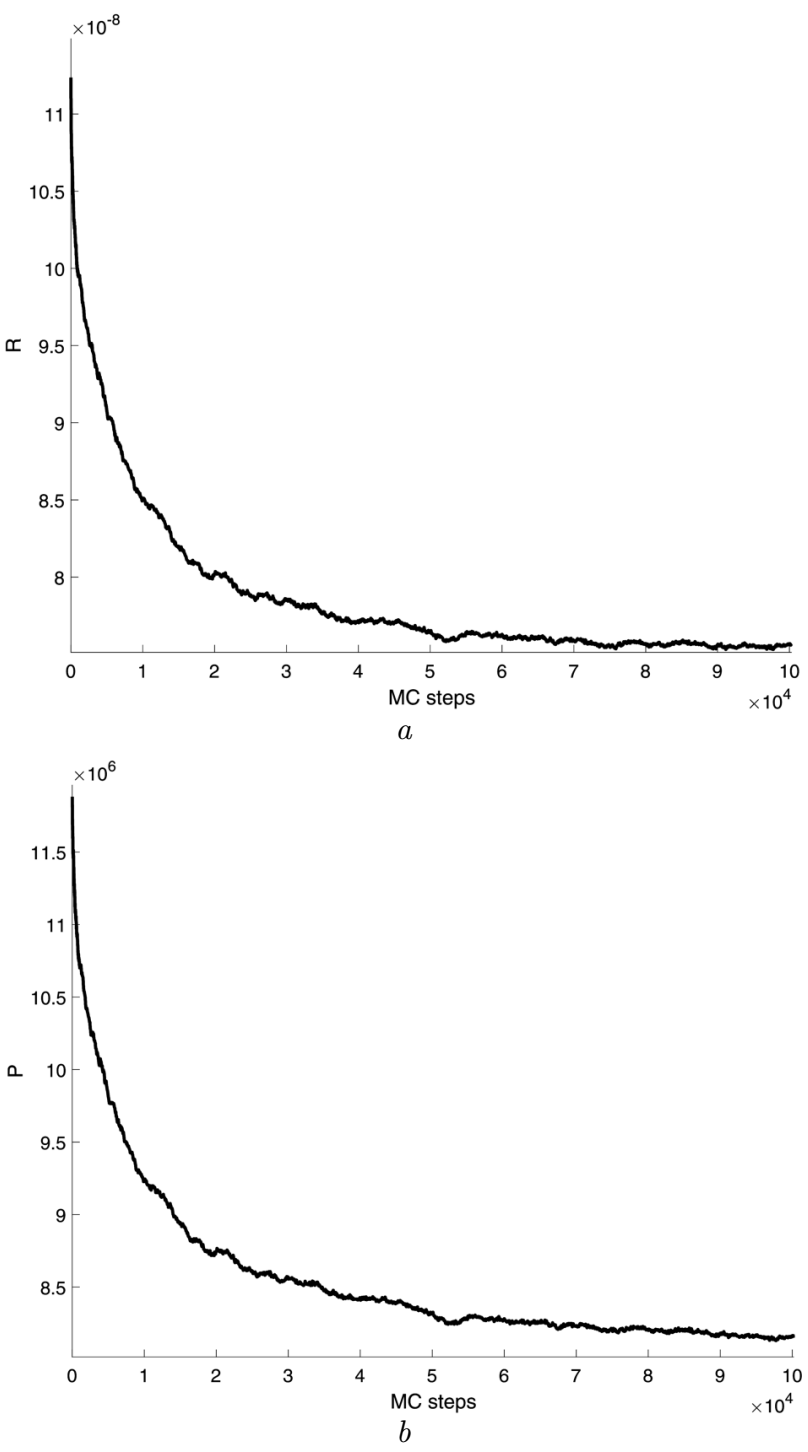

Fig. 5. Dependence of the total resistance $(a)$ and the production of Joule heat $(b)$ at a fixed current and under periodic boundary conditions on atomic flows. $V_{A A} / k_{\mathrm{B}} T=V_{B B} / k_{\mathrm{B}} T=$ $=-1.087, V_{A B} / k_{\mathrm{B}} T=-0.181, l^{\mathrm{el}} / a=0.18$

to grow only in some time. The time dependence of Joule heat generation is shown in Fig. 7.

An attempt to explain the nonmonotonicity of the total resistance in this case is given in Section 5 .

\subsection{Simulation of the evolution \\ of the morphology and the heat generation,} $3 D$ case

Modeling the evolution of a structure in threedimensional samples requires much larger machine

1038

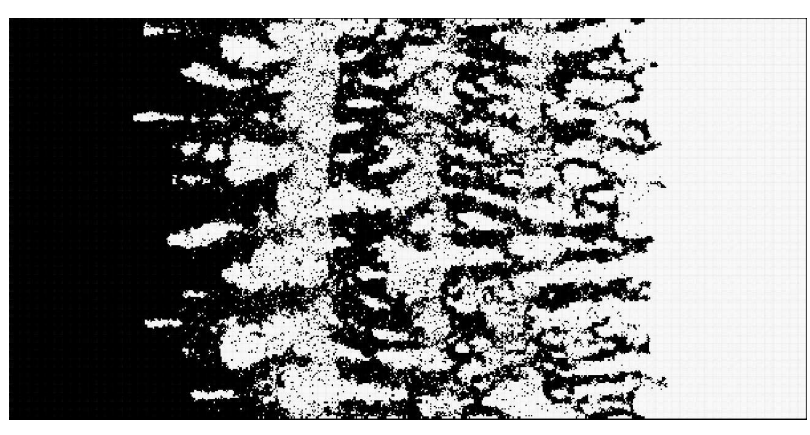

Fig. 6. Morphology at a fixed voltage instead of a fixed current (after 1900 MC steps)

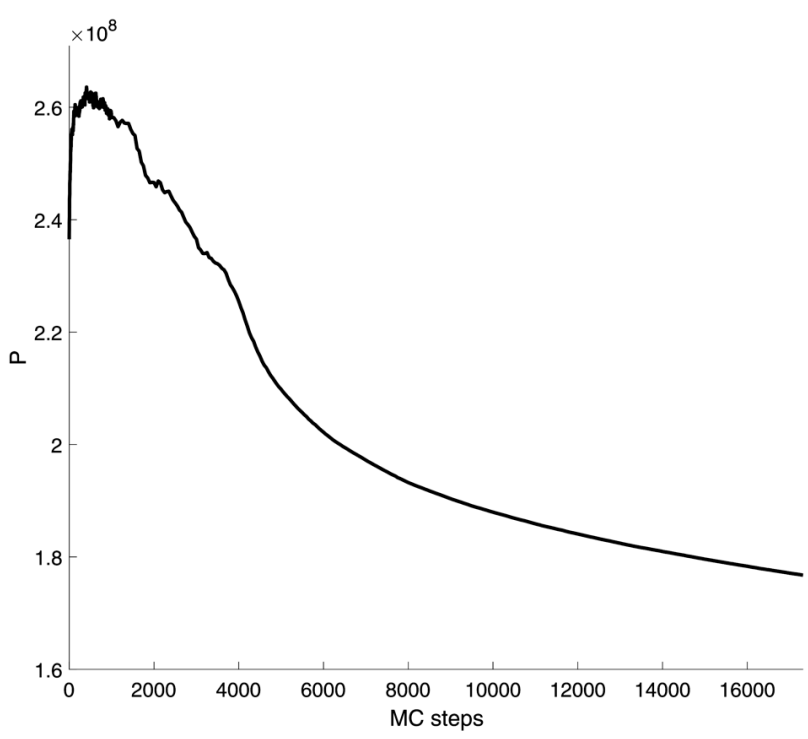

Fig. 7. Time dependence of the heat generation with the fixed voltage $U=3 \mathrm{~V}$

resources, so we give no systematic study, but only a typical example of the evolution. Figure 8 shows the morphology of a three-dimensional decomposable FCC alloy under fixed-voltage conditions. The morphology contains both elements of sequential and parallel connections. Thus, the recurring tendency is revealed in the two-dimensional case.

\section{Discussion}

Starting this study, we expected (as a working hypothesis) to obtain $(a)$ the formation of a parallel connection and a decrease of the resistance in the case of fixed current, $(b)$ the formation of the serial connection and an increase of the resistance in the case of fixed voltage. Then, in both cases, the energy dissipation at the expense of the Joule heat goes to a

ISSN 2071-0194. Ukr. J. Phys. 2017. Vol. 62, No. 12 
minimum. However, our expectations turned out to be somewhat naive.

1. In the case of fixed current, the situation is complicated by the segregation at the ends, which makes the time dependence of the resistance and heat generation nonmonotonic.

2. In the case of a fixed voltage before the stage of growth of the resistance and a drop in the heat dissipation, there is a rather noticeable phase of reducing the resistance and increasing the heat dissipation.

Let us discuss the second feature, by using a simplified model. Our hypothesis is that the decrease of the resistance in the initial stages can be caused not only by the formation of a structure of parallel connection, but also simply by the decomposition, i.e., by the redistribution of components at the local level. To test the hypothesis, we consider firstly two simple phenomenological models.

I. Consider a change in the resistance of the parallel connection of two phases $\alpha$ and $\beta$ during the redistribution of their concentrations with constant phase proportions: the initial concentrations $c_{\alpha}$ and $c_{\beta}$ are equal to 0.5 , and, in the course of the time, $c_{\alpha}$ and $c_{\beta}$ tend to 1 and to zero, respectively. We have $c_{\alpha} p_{\alpha}+c_{\beta} p_{\beta}=\bar{c}$, where $p_{\alpha}$ and $p_{\beta}$ are the volumetric fractions of phases, and $p_{\alpha}+p_{\beta}=1$. In a rough approximation, the resistivity of each phase will be linear in the concentration:

$$
\begin{aligned}
& \rho_{\alpha}\left(c_{\alpha}\right)=\rho_{A} c_{\alpha}+\rho_{B}\left(1-c_{\alpha}\right), \\
& \rho_{\beta}\left(c_{\beta}\right)=\rho_{A} c_{\beta}+\rho_{B}\left(1-c_{\beta}\right) .
\end{aligned}
$$

The average inverse specific resistance of the system, as easily verified, is equal to

$\frac{1}{\bar{\rho}}=\frac{p_{\alpha}}{\rho_{A} c_{\alpha}+\rho_{B}\left(1-c_{\alpha}\right)}+\frac{p_{\beta}}{\rho_{A} c_{\beta}+\rho_{B}\left(1-c_{\beta}\right)}$.

In this case,

$$
\begin{aligned}
& \frac{\partial\left(\frac{1}{\bar{\rho}}\right)}{\partial c_{\alpha}}= \\
& =p_{\alpha} \frac{\left(\rho_{A}-\rho_{B}\right)^{2}\left(c_{\alpha}^{2}-c_{\beta}^{2}\right)\left(\rho_{a}+\rho_{B}\right)}{\left(\rho_{A} c_{\alpha}+\rho_{B}\left(1-c_{\alpha}\right)\right)\left(\rho_{A} c_{\beta}+\rho_{B}\left(1-c_{\beta}\right)\right)}>0 .
\end{aligned}
$$

Thus, as the components are redistributed (when $c_{\alpha}$ increases from 0.5 to 1 ), the inverse resistance increases, and the resistance itself falls, respectively.

II. Consider now a change in the resistance of the serial connection of two phases $\alpha$ and $\beta$ during the

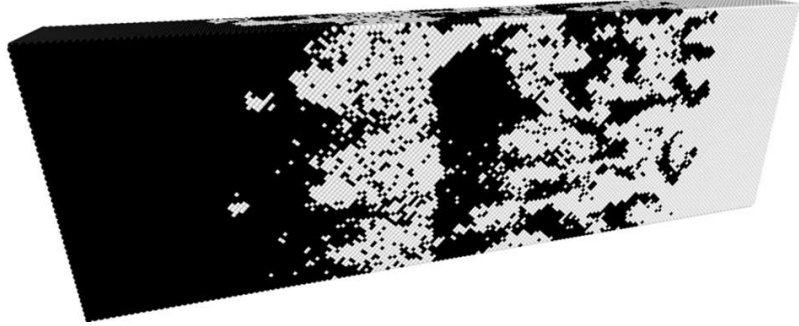

Fig. 8. Morphology at a fixed voltage in the three-dimensional model after 500 Monte Carlo steps

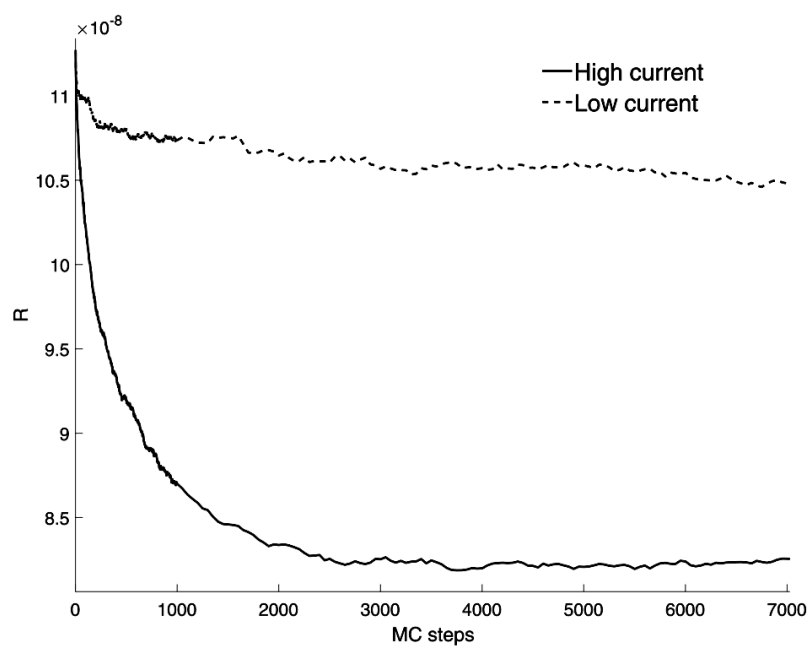

Fig. 9. Dynamics of a change in the complete resistance of the system in the cases of a strong current and its practical absence

redistribution of their concentrations. We have

$\bar{\rho}=p_{\alpha}\left(\rho_{A} c_{\alpha}+\rho_{B}\left(1-c_{\alpha}\right)\right)+$

$+p_{\beta}\left(\rho_{A} c_{\beta}+\rho_{B}\left(1-c_{\beta}\right)\right) \rightarrow \frac{\partial \bar{\rho}}{\partial c_{\alpha}}=0$.

Thus, in the case of a sequential connection, the redistribution of components in a linear approximation for the resistance does not change the total resistance. The real alloy is, as always, an intermediate case between serial and parallel connections, so one can expect that the component stratification should reduce the resistance.

In order to verify the above heuristic reasoning, we compared the evolution of the resistance at the initial stage of decomposition with high and very low currents $\left(2 \times 10^{14} \mathrm{~A} / \mathrm{m}^{2}\right.$ and $\left.2 \mathrm{~A} / \mathrm{m}^{2}\right)$. As shown in Fig. 9, at the initial stage, a decrease of the resistance is observed in both cases. But, in the case of high 


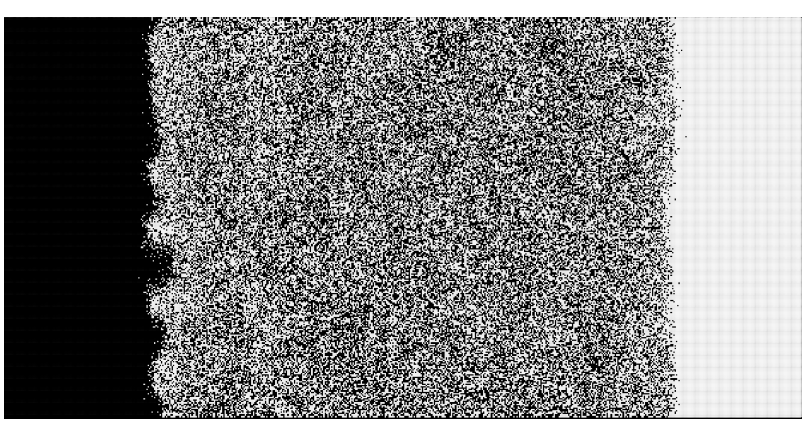

Fig. 10. Morphology of a system that does not undergo the decomposition after 500 Monte Carlo steps. The simulation parameters are similar to the case of fixed current and zero atomic fluxes on the cathode and anode

current, the drop is considerably faster. Thus, in the case of fixed current, two effects (the redistribution of components and the formation of the structure with parallel connection) are observed together. In the case of fixed voltage, we can expect their competition: the stratification of components at the initial stage leads to a decrease of the resistance, but the formation of the structure with serial connection prevails after a while.

The obtained results are consistent with the general property described by I. Prigogine et al. Namely, the production of entropy in a fixed flow tends to decrease. With a fixed force (voltage), it can behave differently.

The results described above indicate a great influence of the electromigration on the evolution of the structure of a two-phase alloy. However, this effect is manifested only in the area of instability of the alloy. To verify this statement, we considered the effect of the electromigration on an alloy with zero mixing energy. The structure of the alloy after 500 steps is shown in Fig. 10. As in the previous cases, the electromigration leads to the segregation of components on the cathode and anode. However, in the intermediate region between the anode and cathode, there are no signs of the parallel or sequential coupling. Thus, even a very strong electric current only helps the system to choose the orientation of the lamellar structure, but does not create this structure in itself.

The authors are grateful to Prof. K.-N. Tu for his idea of entropy production analysis during the electromigration. This work was supported by the Marie Curie International Research Staff Exchange Scheme
Fellowship IRSES within the 7th European Community Framework Programme under Grant 612552; Ministry of Education and Science of Ukraine under Grant 0115 U 000638 and Grant 0117 U 000577.

1. G. Kirchhoff. Über die anwendbarkeit der formeln fur die intensitaten der galvanischen strome in einem systeme linearer leiter auf systeme, die zum theil aus nicht linearen leitern bestehen. Ann. der Physik 151, 189 (1848); H.V. Helmholtz. Über einige gesetze der vertheilung elektrischer strome in korperlichen leitern mit anwendung auf die thierisch-elektrischen versuche. Ann. der Physik 163, 211 (1853); E.T. Jaynes. The minimum entropy production principle. Ann. Rev. of Phys. Chem. 31579 (1980).

2. P.S. Ho, H.B. Huntington. Electromigration and void observation in silver. J. of Phys. and Chem. of Solids 8, 1319 (1966).

3. V.B. Fiks. Ionic Conductivity in Metals and Semiconductors: Electrotransport (Nauka, 1969) (in Russian).

4. Ya.E. Geguzin, M.A. Krivoglaz. Migration of Macroscopic Inclusions in Solids (Consultants Bureau, 1979).

5. P. P. Kuzmenko. Electrotransport, Thermotransport, and Diffusion in Metals (Vyshcha Shkola, 1983) (in Russian).

6. A.M. Gusak, K.N. Tu. Visn. Cherk. Univ. Ser. Fiz.-Mat. Nauk. 117, 12 (2007).

7. P. Glansdorff, I. Prigogine. Thermodynamic Theory of Structure, Stability and Fluctuations (Wiley, 1973).

8. M.A. Ivanov, V.I. Gluschenko. FMM 110, 435 (2010).

9. M.A. Ivanov, V.I. Gluschenko, A.Yu. Naumuk. FMM 113, 3 (2012).

10. A.M. Gusak, T.V. Zaporozhets, Y.O. Lyashenko et al. Diffusion-controlled solid state reactions. In: Alloys, Thin Films, and Nanosystems (Wiley, 2010), Chapt. 10.

11. Handbook of Solid State Diffusion: Vol. 2. Diffusion Analysis in Material Applications, edited by Aloke Paul and Sergiy Divinski (Elsevier, 2017).

12. V.V. Turlo, A.M. Gusak and K.N. Tu. Model of phase separation and of morphology evolution in two-phase alloy. Philos. Magaz. 93, 2013 (2013).

Received 27.08.17

А.М. Гусак, С.В. Марченко, В.В. Турло, О.О. Богатиръов

МОДЕЛЮВАННЯ ВИРОБНИЦТВА ЕНТРОПІї

ТА САМООРГАНІЗАЦІЇ МЕТАЛІЧНОГО СПЛАВУ,

ЩО РОЗПАДАЄТЬСЯ, ПІД ДІЕЮ СИЛЬНОГО

ЕЛЕКТРИЧНОГО СТРУМУ

Р е з ю м е

Синергія розпаду та електроміграції в бінарних сплавах під дією дуже сильного електричного струму проаналізована в рамках підходу швидкості виробництва ентропії і промодельована методом Монте-Карло. Еволюція структури і часова залежність джоулевого нагрівання залежить від того, що саме фіксується під час електроміграції - струм чи напруга.

ISSN 2071-0194. Ukr. J. Phys. 2017. Vol. 62, No. 12 